\title{
Quantification of calcium using localized normalization on laser-induced breakdown spectroscopy data
}

\begin{abstract}
This paper focuses on localized normalization for improved calibration curves in laserinduced breakdown spectroscopy (LIBS) measurements. The calibration curves have been obtained using five samples consisting of different concentrations of calcium (Ca) in potassium bromide (KBr) matrix. The work has utilized Q-switched Nd:YAG laser installed in LIBS2500plus system with fundamental wavelength and laser energy of $650 \mathrm{~mJ}$. Optimization of gate delay can be obtained from signal-to-background ratio (SBR) of Ca II 315.9 and $317.9 \mathrm{~nm}$. The optimum conditions are determined in which having high spectral intensity and SBR. The highest spectral lines of ionic and emission lines of $\mathrm{Ca}$ at gate delay of $0.83 \mu \mathrm{s}$. From SBR, the optimized gate delay is at $5.42 \mu$ s for both Ca II spectral lines. Calibration curves consist of three parts; original intensity from LIBS experimentation, normalization and localized normalization of the spectral line intensity. The R2 values of the calibration curves plotted using locally normalized intensities of Ca I 610.3, 612.2 and 616.2 $\mathrm{nm}$ spectral lines are $0.96329,0.97042$, and 0.96131 , respectively. The enhancement from calibration curves using the regression coefficient allows more accurate analysis in LIBS.
\end{abstract}

Keyword: Laser-induced plasma breakdown; Calcium; Calibration curves; Localized normalization 